Pacific Journal of Mathematics

CONVOLUTIONS OF ARITHMETIC FUNCTIONS OVER 


\title{
CONVOLUTIONS OF ARITHMETIC FUNCTIONS OVER COHESIVE BASIC SEQUENCES
}

\author{
A. A. Gioia and D. L. Goldsmith
}

Necessary and sufficient conditions are given for the generalized convolution of two arithmetic functions which are units with respect to a basic sequence $\mathscr{B}$ to be again a unit on $\mathscr{B}$. These conditions are then investigated from function-theoretic and combinatorial points of view.

1. Introduction. The relation between the multiplicative properties of an arithmetic function and the underlying basic sequence has already been studied in some detail (see [1]). We will confine ourselves here to the consideration of a certain class of basic sequences, and an investigation of the properties of convolutions of arithmetic functions over these basic sequences. The definitions and preliminary results used here may be found in [1], but we will repeat them as a matter of convenience.

A basic sequence $\mathscr{B}$ is a set of pairs $(a, b)$ of positive integers with the properties

$$
\begin{aligned}
& \text { (i) }(1, k) \in \mathscr{B} \quad(k=1,2, \cdots), \\
& \text { (ii) }(a, b) \in \mathscr{B} \text { if and only if }(b, a) \in \mathscr{B}, \\
& \text { (iii) }(a, b c) \in \mathscr{B} \text { if and only if }(a, b) \in \mathscr{B} \text { and }(a, c) \in \mathscr{B} \text {. }
\end{aligned}
$$

Some examples of basic sequences are

$$
\begin{aligned}
\mathscr{S} & =\bigcup_{k=1}^{\infty} S_{k}, \text { where } S_{k}=\{(1, k),(k, 1)\}, \\
\mathscr{C} & =\{(a, b) \mid a \text { and } b \text { are relatively prime positive integers }\}, \\
\mathscr{L} & =\{(a, b) \mid a \text { and } b \text { are any positive integers }\}, \\
\mathscr{T}_{p} & =\mathscr{S} \cup\left\{\left(p^{a}, p^{b}\right) \mid a \text { and } b \text { are any positive integers }\right\},
\end{aligned}
$$

where $p$ is a prime.

A pair $(a, b)$ of positive integers is called a primitive pair if both $a$ and $b$ are primes. If $a \neq b$, the pair is a type $I$ primitive pair; if $a=b$, the pair is a type $I I$ primitive pair. We see that a basic sequence is completely determined by the primitive pairs it contains. If $\Phi$ is any set of pairs (primitive or not) of positive integers, the basic sequence generated by $\Phi$ is defined to be

$$
\Gamma[\Phi]=\bigcap \mathscr{D},
$$

where the intersection is taken over all basic sequences $\mathscr{D}$ which contain $\Phi$. Thus, a basic sequence is generated by its subset of 
primitive pairs. An arithmetic function is a real-valued function defined on the set of natural numbers. Let $f$ be an arithmetic function and let $m$ and $n$ be natural numbers. We define

$$
\alpha_{f}(m, n)=\left\{\begin{array}{cl}
\frac{f(m) f(n)-f(m n)}{|f(m) f(n)|+|f(m n)|} & \text { if }|f(m) f(n)|+|f(m n)|>0, \\
0 & \text { if } f(m) f(n)=f(m n)=0 .
\end{array}\right.
$$

Let $\mathscr{B}$ be a basic sequence. We say that the index of multiplicativity of $f$ with respect to $\mathscr{B}$ exists, and has the value $I(f, \mathscr{B})$, provided

$$
\lim _{k \rightarrow \infty} \alpha_{f}\left(m_{k}, n_{k}\right)=I(f, \mathscr{B})
$$

for every collection of pairs $\left\{\left(m_{k}, n_{k}\right)\right\}_{k=1}^{\infty}$ in $\mathscr{B}$ for which $\lim _{k \rightarrow \infty} m_{k} n_{k}=\infty$.

$f$ is said to be multiplicative with respect to $\mathscr{B}$ if $f(m) f(n)=$ $f(m n)$ for every pair $(m, n)$ in $\mathscr{B}$. Functions which are multiplicative with respect to $\mathscr{C l}$ are multiplicative in the usual sense. For $f$ to be multiplicative with respect to $\mathscr{B}$, it is clearly necessary that $I(f, \mathscr{B})=0$.

If $\mathscr{B}$ is a basic sequence and $f$ is an arithmetic function such that

(1) $I(f, \mathscr{B})=0$,

(2) there exists an integer $N$ such that $f(k) \neq 0$ and $f(k)$ does not change sign for $k \geqq N$,

then $f$ is called a unit with respect to $\mathscr{B}$. A unit is positive or negative depending on the sign of $f(k)$ for large values of $k$.

The present investigation was motivated by an attempt to solve the following problem: Characterize those basic sequences $\mathscr{B}$ for which, given any pair of units $f, g$ on $\mathscr{B}$ and any basic sequence $\mathscr{E}$ containing $\mathscr{B}$, the convolution $f \circ_{\mathscr{E}} g$ is again a unit on $\mathscr{B}$, where

$$
\left(f \circ \circ_{\mathscr{E}} g\right)(n)=\sum_{(d, n / d) \in \mathscr{E}} f(d) g(n / d) .
$$

In view of the following lemma we need consider only basic sequences $\mathscr{B}$ which are contained in $\mathscr{l}$.

LEMmA 1.1. If $\mathscr{B} \not \subset \mathscr{M}$, then there are units $f, g$ on $\mathscr{B}$ and $a$ basic sequence $\mathscr{E}$ which contains $\mathscr{B}$ such that $f \circ \mathscr{E} g$ is not a unit on $\mathscr{B}$.

For if $\mathscr{B} \not \subset \mathscr{C}$, then $\mathscr{B}$ contains some type II primitive pair, say $(p, p)$. Take $\mathscr{E}=\mathscr{B}$ and let $f(n)=g(n)=1(n=1,2, \cdots)$. Then $f$ and $g$ are units on $\mathscr{B}$, but $\left(f \circ \circ_{\mathscr{\varepsilon}} g\right)\left(p^{t}\right)=t+1$, so $I\left(f \circ_{\mathscr{\&}} g, \mathscr{B}\right)$ does not exist. 
A basic sequence $\mathscr{B}$ is said to be cohesive provided that for each positive integer $k$ there is an integer $a>1$ such that $(a, k) \in \mathscr{B}$. The characterization required above is given by

THEOREM 1.2. Suppose $\mathscr{B} \subset \mathscr{A}$. In order that $f \circ \mathscr{\&} g$ be a unit on $\mathscr{B}$ for every pair $f, g$ of units on $\mathscr{B}$ and every basic sequence $\mathscr{E}$ containing $\mathscr{B}$, it is both necessary and sufficient that $\mathscr{B}$ be cohesive.

The following example will illustrate Theorem 1.2 and some of the preceding definitions.

EXAMPLE 1.3. Let $\left\{p_{j}\right\}_{j=1}^{\infty}$ be an enumeration of the primes in ascending order, and let $\mathscr{B}$ be the basic sequence generated by the following set of primitive pairs:

$$
\bigcup_{j=1}^{\infty}\left\{\bigcup_{i=1}^{\infty}\left(p_{j}, p_{j+1+i}\right)\right\} \text {. }
$$

Thus $\mathscr{B}$ is generated by the pairs $(2.5),(2,7),(2,11), \cdots,(3,7),(3,11)$, $(3,13), \cdots$, and so forth. $\mathscr{B} \subset \mathscr{C l}$ and $\mathscr{B}$ is cohesive. Now define the arithmetic functions $f$ and $g$ by

$$
\begin{aligned}
& f(k)=\left\{\begin{array}{l}
1 \text { if } 6 \nmid k, \\
2 \text { if } 6 \mid k,
\end{array}\right. \\
& g(k)= \begin{cases}k \text { if } 6 \nmid k, \\
m \text { if } k=6^{a} m, \text { where } 6 \nmid m .\end{cases}
\end{aligned}
$$

$f$ and $g$ are units on $\mathscr{B}$, and $f \circ_{\mathscr{C}} g$ is also a unit on $\mathscr{B}$ for every basic sequence $\mathscr{E}$ which contains $\mathscr{B}$.

We note that although neither $f$ nor $g$ is multiplicative with respect to $\mathscr{C}$, each is multiplicative with respect to $\mathscr{B}$. We will see shortly (Theorem 2.3) that the multiplicativity of $f$ and $g$ on $\mathscr{B}$ was unavoidable.

Theorem 1.2 provides a link between the multiplicative properties of certain arithmetic functions and the combinatorial properties (e.g., cohesiveness) of the underlying basic sequence. We discuss the function-theoretic consequences of cohesiveness in $\S 2$ and use the results obtained there to give a proof of Theorem 1.2 in $\S 3$.

In $\S 4$ we examine the combinatorical aspects of cohesiveness and in $\S 5$ we present two examples which supplement the results given in $\S 4$.

2. Function-theoretic properties of cohesiveness. From the statement of Theorem 1.2 one is led naturally to look for other characterizations of cohesiveness in terms of the behavior of the units 
on A particularly simple criterion is given by

THEOREM 2.1. A basic sequence $\mathscr{B}$ is cohesive if and only if, for every unit $f$ on $\mathscr{B}, f(k)>0$ for all $k$.

Proof. Assume that $\mathscr{B}$ is cohesive and let a unit $f$ on $\mathscr{B}$ and a positive integer $k$ be given. Since $(1, k) \in \mathscr{B}$ and since there exists an integer $a>1$ such that $(a, k)=(a, 1 \cdot k) \in \mathscr{B}$, it follows from [1, Theorem 4.11(a)] that $f(k)>0$.

Conversely, suppose that $\mathscr{B}$ is not cohesive. Then there is an integer $k_{0}$ such that, for every $a>1,\left(a, k_{0}\right) \notin \mathscr{B}$. Note that $k_{0}>1$. Consider the function $f$ defined by $f(n)=1$ if $n \neq k_{0}, f\left(k_{0}\right)=-1$. It is sufficient to show that $f$ is a unit on $\mathscr{B}$.

Suppose $(u, v) \in \mathscr{B}, u v>k_{0}$. If either $u=k_{0}$ or $v=k_{0}$, say $v=k_{0}$, then $(u, v)=\left(u, k_{0}\right) \in \mathscr{B}$. That implies $u=1$, so $u v=k_{0}$. But this contradicts the assumption $u v>k_{0}$. So $u \neq k_{0}$ and $v \neq k_{0}$, hence $f(u)=f(v)=1$ and $\alpha_{f}(u, v)=0$. It follows that $I(f, \mathscr{B})=0$ and $f$ is a unit on $\mathscr{B}$.

Note that the exact value of $f\left(k_{0}\right)$ never comes into play in the second part of the proof of the preceding theorem. In fact, we have actually proved

THEOREM 2.2. If $\mathscr{B}$ is not cohesive there exists an integer $k_{0}>1$ (which depends only on $\mathscr{B}$ ) such that, for any given value $V$, there is a unit $f$ on $\mathscr{B}$ satisfying $f\left(k_{0}\right)=V$.

The following necessary condition for cohesiveness is an immediate consequence of [1, Theorem 4.11].

THEOREM 2.3. If $\mathscr{B}$ is a cohesive basic sequence, then every unit on $\mathscr{B}$ is multiplicative with respect to $\mathscr{B}$.

COROLlary 2.3.1. If, for every unit $f$ on $\mathscr{S}, f(k)>0$ for all $k$, then every unit on $\mathscr{B}$ is multiplicative with respect to $\mathscr{S}$.

The converse of Theorem 2.3 is not true; simply take $\mathscr{B}=\mathscr{S}$.

Theorem 2.1 illustrates the following line of reasoning: Suppose $f$ has some multiplicative properties with respect to a basic sequence $\mathscr{B}$ and that $f(k)$ satisfies certain conditions for large values of $k$. Then if $\mathscr{B}$ is large enough, one might expect $f$ to satisfy these conditions for all values of $k$. Another example is given by

THEOREM 2.4. For any basic sequence $\mathscr{B}$, the following two statements are equivalent:

(1) $\mathscr{B}$ is cohesive; 
(2) every function $f$ which satisfies

(a) $I(f, \mathscr{B})=0$,

(b) $f(k) \neq 0$ for $k \geqq N$,

also satisfies $f(k) \neq 0$ for all $k$.

Proof. Suppose $\mathscr{B}$ is cohesive and that $f$ satisfies (a) and (b). Since $I(f, \mathscr{B})=0$ we may apply Lemma 2.3 of [1]. Let $N_{1}$ be the integer given there and let $N^{*}=\max \left\{N, N_{1}\right\}$. For any positive integer $n$, the cohesiveness of $\mathscr{B}$ implies the existence of an integer $a>1$ such that $(a, n) \in \mathscr{B}$. If $t$ is chosen so that $a^{t} \geqq N^{*}$, then $a^{t} n \geqq N$ and so $f\left(a^{t} n\right) \neq 0$. But also $a^{t} n \geqq N_{1}$, and since $\left(\mathrm{a}^{t}, n\right) \in \mathscr{B}$, Lemma 2.3 of [1] implies $f\left(a^{t}\right) f(n) \neq 0$. Hence $f(n) \neq 0$ and (2) holds.

On the other hand, suppose $\mathscr{B}$ is not cohesive. Then by Theorem 2.2 (with $V=0$ ), there is a unit $f$ on $\mathscr{B}$ such that $f\left(k_{0}\right)=0$. So (2) is false.

3. Proof of Theorem 1.2. Suppose first that $\mathscr{B}$ is cohesive. By Theorem 2.3, the units $f$ and $g$ are multiplicative with respect to $\mathscr{B}$. Hence, by Theorem 1 in [2], $f \circ_{\mathscr{B}} g$ is also multiplicative with respect to $\mathscr{B}$. Moreover, by Theorem 2.1, $f(k)$ and $g(k)$ are positive for all values of $k$, so $(f \circ g)(k)$ is positive for every $k$. Therefore $f \circ_{\mathscr{C}} g$ is a unit on $\mathscr{B}$.

If $\mathscr{B}$ is not cohesive, then there is an integer $k_{0}>1$ such that $\left(a, k_{0}\right) \notin \mathscr{B}$ for each $a>1$. Let $p_{0}$ be any prime which does not divide $k_{0}$ and define $\mathscr{E}$ to be the basic sequence generated by $\mathscr{B} \cup\left(p_{0}, k_{0}\right)$ :

$$
\mathscr{E}=\Gamma\left[\mathscr{B} \cup\left(p_{0}, k_{0}\right)\right]
$$

Since $\left(p_{0}, k_{0}\right) \notin \mathscr{B}$ and since $p_{0}$ and $k_{0}$ are relatively prime, we have $\mathscr{B} \subset \mathscr{E} \subset \mathscr{M}$.

We will show that there are units $f, g$ on $\mathscr{B}$ for which $f \circ_{\mathscr{f}} g$ is not a unit on $\mathscr{B}$. In fact, let $g(n)=1$ for all $n$ and define $f$ by

$$
f(n)=\left\{\begin{array}{l}
1 \text { if } n \neq k_{0}, \\
1-2^{\sharp} E_{k_{0}} \text { if } n=k_{0},
\end{array}\right.
$$

where ${ }^{\sharp} E_{m}$ is the number of pairs $(a, b)$ in $\mathscr{E}$ for which $a b=m$.

Clearly $g$ is a unit. The proof that $f$ is a unit on $\mathscr{B}$ is essentially the second part of the proof of Theorem 2.1. To show that $f \circ g$ is not a unit with respect to $\mathscr{B}$, we will show that

$$
(f \circ \mathscr{\mathscr { C }} g)\left(p_{0}^{t} k_{0}\right)=\sum_{\substack{d \mid p_{0}^{t} k_{0} \\\left(d, p_{0}^{t} k_{0} / d\right) \in \mathscr{\&}}} f(d) g\left(p_{0}^{t} k_{0} / d\right)
$$


is zero for every $t \geqq 1$. We note first that if $d=p_{0}^{s} l$ where $l \mid k_{0}$, then either $s=0$ or $s=t$ since $\mathscr{E} \subset \mathscr{A}$. Hence

$$
\left(f \circ \circ_{\mathscr{E}} g\right)\left(p_{0}^{t} k_{0}\right)=\sum_{\substack{l \mid k_{0} \\\left(l, k_{0} p_{0}^{t} / l\right) \in \mathscr{\varepsilon}}} f(l)+\sum_{\substack{l \mid k_{0} \\\left(p_{0}^{t} l, k_{0} / l\right) \in \mathscr{\varepsilon}}} f\left(p_{0}^{t} l\right) .
$$

Now $\left(p_{0}, k_{0}\right) \in \mathscr{E}$, so $\left(l, p_{0}^{t}\right) \in \mathscr{E}$ for every $l$ which divides $k_{0}$. Hence

$$
\left(l, \frac{k_{0}}{l} p_{0}^{t}\right) \in \mathscr{E} \Longleftrightarrow\left(l, \frac{k_{0}}{l}\right) \in \mathscr{E} .
$$

Also, $\left(p_{0}^{t}, k_{0} / l\right) \in \mathscr{E}$ for every $l$ which divides $k_{0}$, so

$$
\left(p_{0}^{t} l, \frac{k_{0}}{l}\right) \in \mathscr{E} \Longleftrightarrow\left(l, \frac{k_{0}}{l}\right) \in \mathscr{E} \text {. }
$$

It follows that

$$
\begin{aligned}
\left(f \circ \circ_{\mathscr{E}} g\right)\left(p_{0}^{t} k_{0}\right) & =\sum_{\substack{l \mid k_{0} \\
\left(l, k_{0} l l\right) \in \mathscr{E}}} f(l)+\sum_{\substack{l \mid k_{0} \\
\left(l, k_{0} l l\right) \in \mathscr{E}}} f\left(p_{0}^{t} l\right) \\
& =f\left(k_{0}\right)+\sum_{\substack{l \mid k_{0}, l<k_{0} \\
\left(l, k_{0} l l\right) \in \mathscr{E}}} 1+\sum_{\substack{l \mid k_{0} \\
\left(l, k_{0} / l\right) \in \mathscr{E}}} 1 \\
& =f\left(k_{0}\right)+\left({ }^{\sharp} E_{k_{0}}-1\right)+{ }^{\sharp} E_{k_{0}}=0 .
\end{aligned}
$$

Thus $f \circ_{\mathscr{E}} g$ is not a unit on $\mathscr{B}$, and the proof of Theorem 1.2 is complete.

An arithmetic function $f$ is said to have nonexceptional index with respect to a basic sequence $\mathscr{B}$ if $I(f, \mathscr{B})$ exists and $0<|I(f, \mathscr{B})|<1$. Using the fact that $f$ has nonexceptional index if and only if $f=F f^{*}$, where $f^{*}$ is a unit on $\mathscr{B}$ and $F$ is a constant satisfying $F>0, F \neq 1$ (see Theorem 5.1 in [1]), we have the following straightforward consequence of Theorem 1.2.

TheOREM 3.1. Let $\mathscr{B}$ and $\mathscr{E}$ be basic sequences such that $\mathscr{B}$ is cohesive and $\mathscr{B} \subset \mathscr{E} \cap \mathscr{M}$. If $f$, and also $g$, is either a unit on $\mathscr{B}$ or has nonexceptional index, then

(1) $f \circ_{\mathscr{g}} g=H h^{*}$ for some unit $h^{*}$ on $\mathscr{B}$ and some constant $H>0$,

(2) $f \circ_{\mathscr{\&}} g$ is either a unit or has nonexceptional index with respect to $\mathscr{B}$,

(3) $I\left(f \circ \circ_{\mathscr{E}} g, \mathscr{B}\right)=\frac{I(f, \mathscr{B})+I(g, \mathscr{B})}{1+I(f, \mathscr{B}) I(g, \mathscr{B})}$.

4. Combinatorial properties of cohesiveness. We will use the following notation:

$$
P=\text { set of all primes, }
$$




$$
\begin{aligned}
& C_{\mathscr{B}}(m)=\{p \mid p \in P,(p, m) \in \mathscr{B}\}, \\
& C_{\mathscr{B}}(A)=\bigcup_{a \in A} C_{\mathscr{B}}(a) .
\end{aligned}
$$

A basic sequence which is cohesive is one which, roughly speaking, has enough primitive pairs and for which the primitive pairs are properly distributed. The following two theorems and their corollaries will make these notions more precise.

TheOREM 4.1. If $\mathscr{B}$ is cohesive, then

$$
C_{\mathscr{S}}\left(C_{\mathscr{B}}(p)\right)=P
$$

for every prime p.

Proof. Suppose $s \in P$. Since $\mathscr{B}$ is cohesive, there is a prime $q_{0}$ such that $\left(q_{0}, s p\right) \in \mathscr{B}$. But this means $\left(s, q_{0}\right) \in \mathscr{B}$ and $\left(q_{0}, p\right) \in \mathscr{B}$. Hence

$$
s \in C_{\mathscr{B}}\left(q_{0}\right) \subset \bigcup_{q \in C_{\mathscr{B}}(p)} C_{\mathscr{B}}(q)=C_{\mathscr{B}}\left(C_{\mathscr{S}}(p)\right)
$$

We recall that a prime $p$ is said to have property $F$ with respect to the basic sequence $\mathscr{B}$ if $p$ is finitely distributed with respect to $\mathscr{B}$ (i.e., $C_{\mathscr{B}}(p)$ is finite) and if every prime $q$ in $C_{\mathscr{S}}(p)$ is also finitely distributed with respect to $\mathscr{B}$.

COROLLARY 4.1.1. If $\mathscr{B}$ is cohesive, then no prime has property $F$ with respect to $\mathscr{B}$.

COROLLARY 4.1.2. If $\mathscr{B}$ is cohesive, then $\mathscr{B}$ must contain infinitely many type I primitive pairs.

CoROLlaRY 4.1.3. Suppose $\mathscr{B}$ is cohesive. If there is a prime $p_{0}$ for which there is only one prime $q$ such that $\left(q, p_{0}\right) \in \mathscr{B}$, then $(q, r) \in \mathscr{B}$ for every prime $r$.

THEOREM 4.2. If $\mathscr{B}$ is a cohesive basic sequence, then either $C_{\mathscr{B}}(p)$ is infinite for every prime $p$, or there is a prime $q$ for which $C_{\mathscr{B}}(q)=P$.

CoRollary 4.2.1. If $\mathscr{B}$ is cohesive and $\mathscr{B} \subset \mathscr{A l}$, then $C_{\mathscr{B}}(p)$ is infinite for every prime $p$.

Proof. We first note the obvious facts that (i) for any basic sequence $\mathscr{B}$, if $m \mid n$ then $C_{\mathscr{B}}(m) \supset C_{\mathscr{B}}(n)$ and (ii) if $\mathscr{B}$ is cohesive, then $C_{\mathscr{B}}(m)$ is not empty for any $m$. 
Suppose $C_{0}\left(p_{0}\right)$ is finite for some prime $p_{0}$. Enumerate the sequence of all primes: $p_{1}, p_{2}, \cdots$. Then

$$
C_{\mathscr{S}}\left(p_{0}\right) \supset C_{\mathscr{s}}\left(p_{0} p_{1}\right) \supset C_{. 今}\left(p_{0} p_{1} p_{2}\right) \supset \cdots .
$$

We thus have a nested, nonincreasing sequence of nonempty finite sets of primes, hence

$$
A=\bigcap_{K=1}^{\infty} C_{\mathscr{O}}\left(\prod_{j=0}^{K} p_{j}\right)
$$

is not empty. Every prime $q$ in $A$ satisfies $\left(q, p_{j}\right) \in \mathscr{B}, j=1,2, \cdots$. That proves the theorem.

The corollary follows from the fact that if $C_{.3}(q)=P$ for some prime $q$, then $(q, q) \in \mathscr{B}$, so $\mathscr{B}$ cannot be contained in $\mathscr{A}$.

5. Some examples. The theorems in the preceding section give us information (in terms of the primitive pairs in $\mathscr{B}$ ) about the size and structure of a cohesive basic sequence. The following two examples suggest that the structure of the primitive pairs in $\mathscr{B}$ is more important than the number of primitive pairs in determining whether $\mathscr{B}$ is cohesive.

We recall that the density of a basic sequence $\mathscr{B}$ is defined to be

$$
\delta(\mathscr{B})=\lim _{N \rightarrow \infty} \frac{1}{N} \sum_{k=1}^{N} \frac{\# B_{k}}{d(k)},
$$

where ${ }^{\sharp} B_{k}$ is the number of pairs $(a, b)$ in $\mathscr{B}$ for which $a b=k$, and $d(k)$ is the number of divisors of $k$.

EXAMPle 5.1. The basic sequence $\mathscr{B}$ generated by $\{(2, p) \mid p \in P\}$ is cohesive and $\delta(\mathscr{B})=0$. Thus a cohesive basic sequence may be very small if it contains the "right" primitive pairs.

ExAmPLE 5.2. Let $P_{1}$ and $P_{2}$ be two disjoint infinite sets of primes such that $P_{1} \cup P_{2}=P$, and let $\mathscr{B}$ be generated by $\left\{(p, q) \mid p, q \in P_{1}\right.$ or $\left.p, q \in P_{2}\right\}$. Clearly $\mathscr{B}$ is not cohesive. Moreover, by choosing the primes in $P_{2}$ to be large and very sparse, we can make $\delta(\mathscr{B})$ as close to 1 as we wish. So $\mathscr{B}$ can be very large, but without a suitable structure on the set of primitive pairs cohesiveness cannot be guaranteed.

We note that no prime is finitely distributed with respect to $\mathscr{B}$ and that $C_{\mathscr{S}}(p)$ is infinite for every prime $p$, hence the converses of Corollary 4.1.1 and Theorem 4.2 do not hold. 


\section{REFERENCES}

1. D. L. Goldsmith, On the multiplicative properties of arithmetic functions, Pacific J. Math., 27 (1968), 283-304.

2. — A generalized convolution for arithmetic functions, Duke J. Math., 38 (1971), 279-283.

Received March 31, 1970.

WESTERN MICHIGAN UNIVERSITY 



\section{PACIFIC JOURNAL OF MATHEMATICS}

\section{EDITORS}

\author{
H. SAMELSON \\ Stanford University \\ Stanford, California 94305 \\ C. R. HовBY \\ University of Washington \\ Seattle, Washington 98105
}

J. DUGUndis

Department of Mathematics

University of Southern California

Los Angeles, California 90007

RICHARD ARENS

University of California

Los Angeles, California 90024

\section{ASSOCIATE EDITORS}
E. F. BeCKenbaCH
B. H. NeUmanN
F. WOLF
K. YoshidA

\section{SUPPORTING INSTITUTIONS}

\author{
UNIVERSITY OF BRITISH COLUMBIA \\ CALIFORNIA INSTITUTE OF TECHNOLOGY \\ UNIVERSITY OF CALIFORNIA \\ MONTANA STATE UNIVERSITY \\ UNIVERSITY OF NEVADA \\ NEW MEXICO STATE UNIVERSITY \\ OREGON STATE UNIVERSITY \\ UNIVERSITY OF OREGON \\ OSAKA UNIVERSITY \\ UNIVERSITY OF SOUTHERN CALIFORNIA
}

\author{
STANFORD UNIVERSITY \\ UNIVERSITY OF TOKYO \\ UNIVERSITY OF UTAH \\ WASHINGTON STATE UNIVERSITY \\ UNIVERSITY OF WASHINGTON

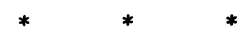 \\ AMERICAN MATHEMATICAL SOCIETY \\ CHEVRON RESEARCH CORPORATION \\ NAVAL WEAPONS CENTER
}

The Supporting Institutions listed above contribute to the cost of publication of this Journal, but they are not owners or publishers and have no responsibility for its content or policies.

Mathematical papers intended for publication in the Pacific Journal of Mathematics should be in typed form or offset-reproduced, (not dittoed), double spaced with large margins. Underline Greek letters in red, German in green, and script in blue. The first paragraph or two must be capable of being used separately as a synopsis of the entire paper. The editorial "we" must not be used in the synopsis, and items of the bibliography should not be cited there unless absolutely necessary, in which case they must be identified by author and Journal, rather than by item number. Manuscripts, in duplicate if possible, may be sent to any one of the four editors. Please classify according to the scheme of Math. Rev. Index to Vol. 39. All other communications to the editors should be addressed to the managing editor, Richard Arens, University of California, Los Angeles, California, 90024.

50 reprints are provided free for each article; additional copies may be obtained at cost in multiples of 50 .

The Pacific Journal of Mathematics is published monthly. Effective with Volume 16 the price per volume (3 numbers) is $\$ 8.00$; single issues, $\$ 3.00$. Special price for current issues to individual faculty members of supporting institutions and to individual members of the American Mathematical Society: $\$ 4.00$ per volume; single issues $\$ 1.50$. Back numbers are available.

Subscriptions, orders for back numbers, and changes of address should be sent to Pacific Journal of Mathematics, 103 Highland Boulevard, Berkeley, California, 94708.

PUBLISHED BY PACIFIC JOURNAL OF MATHEMATICS, A NON-PROFIT CORPORATION

Printed at Kokusai Bunken Insatsusha (International Academic Printing Co., Ltd.), 7-17, Fujimi 2-chome, Chiyoda-ku, Tokyo, Japan. 


\section{Pacific Journal of Mathematics}

Vol. 38, No. $2 \quad$ April, 1971

Richard Davis Anderson and Thomas Ashland Chapman, Extending

homeomorphisms to Hilbert cube manifolds .................. 281

Nguyen Huu Anh, Restriction of the principal series of $\operatorname{SL}(n, \mathbf{C})$ to some

reductive subgroups................................ 295

David W. Boyd, Indices for the Orlicz spaces . . . . . . . . . . . . 315

William Garfield Bridges, The polynomial of a non-regular digraph ...... 325

Billie Chandler Carlson, Robert K. Meany and Stuart Alan Nelson, Mixed

arithmetic and geometric means........................ 343

H. A. Çelik, Commutative associative rings and anti-flexible rings ...... 351

Hsin Chu, On the structure of almost periodic transformation groups ...... 359

David Allyn Drake, The translation groups of n-uniform translation

Hjelmslev planes ................................ 365

Michael Benton Freeman, The polynomial hull of a thin two-manifold . . . . 377

Anthony Alfred Gioia and Donald Goldsmith, Convolutions of arithmetic

functions over cohesive basic sequences .................... 391

Leslie C. Glaser, A proof of the most general polyhedral Schoenflies

conjecture possible ................................

Thomas Lee Hayden and Ted Joe Suffridge, Biholomorphic maps in Hilbert

space have a fixed point ................................ 419

Roger Alan Horn, Schlicht mappings and infinitely divisible kernels ...... 423

Norman Ray Howes, On completeness ...................... 431

Hideo Imai, Sario potentials on Riemannian spaces................ 441

A. A. Iskander, Subalgebra systems of powers of partial universal

algebras.

Barry E. Johnson, Norms of derivations of $\mathscr{L}(\mathrm{X})$.

David Clifford Kay and Eugene W. Womble, Axiomatic convexity theory and relationships between the Carathéodory, Helly, and Radon numbers

Constantine G. Lascarides, A study of certain sequence spaces of Maddox

and a generalization of a theorem of Iyer .............

C. N. Linden, On Blaschke products of restricted growth .

John S. Lowndes, Some triple integral equations ................. 515

Declan McCartan, Bicontinuous preordered topological spaces ......... 523

S. Moedomo and J. Jerry Uhl, Jr., Radon-Nikodým theorems for the Bochner and Pettis integrals ...

Calvin Cooper Moore and Joseph Albert Wolf, Totally real representations

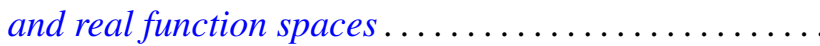

Reese Trego Prosser, A form of the moment problem for Lie groups. ... 